\title{
Bone blood flow is influenced by muscle contractions
}

\author{
Jan Erik Näslund ${ }^{1}$, Sofie Näslund ${ }^{2}$, Erik Lundeberg ${ }^{1}$, Lars-Göran Lindberg ${ }^{3}$, Iréne Lund ${ }^{1}$ \\ ${ }^{1}$ Department of Physiology and Pharmacology, Karolinska Institutet, Stockholm, Sweden; \\ ${ }^{2}$ Gävle Hospital, Gävle, Sweden; \\ ${ }^{3}$ Department of Biomedical Engineering, Linköping University, Linköping, Sweden. \\ Email: Jan.E.Naslund@ki.se; s_n_a_s@hotmail.com; erik_lundeberg@hotmail.com; larli@imt.liu.se; Irene.Lund@ki.se
}

Received 18 April 2011; revised 11 May 2011; accepted 27 May, 2011.

\begin{abstract}
Forces acting on the skeleton could be divided into those originating from gravitational loading and those originating from muscle loading. Flat bones in a non-weight-baring segment of the skeleton probably experience forces mostly generated by muscle contractions. One purpose of muscle contractions is to generate blood flow within skeletal tissues. The present study aimed to investigate the pulsatile patellar bone blood flow after low and high intensity leg extension exercises. Forty-two healthy individuals volunteered for the study. Dynamic isotonic one leg extension/flexion exercises were performed in a leg extension machine. Randomly, the exercises were performed with the left or right leg with either 10 repetition maximum (10 RM) continuously without any resting periods (high intensity muscle work), or 20 RM with a 2 second rest between contractions (low intensity muscle work). The work load, expressed in kilograms totally lifted, was identical in both legs. The pulsatile patellar blood flow was recorded continuously using a photoplethysmographic technique. Blood pressure was measured continuously during muscle work by a non-invasive method (Finapress). The patellar pulsatile bone blood flow increased significantly more after high intensity muscle work (61\%) compared to the same work load performed using a lower intensity $(22 \%), p=0.000073$. Systolic blood pressure changed equally during and after both interventions. Post-exercise bone hyperaemia appears to be correlated to the intensity of muscle contractions in the muscle compartment attached to the bone.
\end{abstract}

Keywords: Bone; Blood Flow; Blood Pressure; Photoplethysmography

\section{INTRODUCTION}

It is generally concluded that weight-baring bones grow and are remodeled in response to mechanical loading. It is however not totally agreed on how forces acting on bone cells influence bone morphology (mechanotransduction), but the importance of solute transport for cellular mechanotransduction [1] and cellular excitation due to interstitial fluid flow (IFF) have been implicated [2].

The contribution of strain in terms of magnitude, rate, cycles and unusual distribution, is still a matter of debate [3]. Also, the originating source of loading like gravitational loading and muscle loading have both been proven influencing different aspects of skeletal morphology [4-5], while it has been discussed which type of loading is the most important for bone health [6]. The relative contribution of each type will most likely depend on the specific activity, the location of the bone in question within the skeleton, and whether the bone is weightbaring or not [2]. In humans, gravitational loading acts predominantly on the lower limb and on the axial skeleton. Non-weight bearing bones like scapula, ribs, cranial bones, and patella are also exposed to bone resorption, modeling, and remodeling. A flat bone in a non-weight baring segment of the skeleton is probably exposed to forces mostly generated by muscle contractions. The function of these muscle contractions may, in addition to resisting the force of gravity and inducing locomotion, also be to generate increase of blood flow within skeletal tissues. It has even been speculated that a co-dependence of muscle and bone exists, and that muscle is not only a source of mechanical stimuli for bone but also serves as an important endocrine organ that may indirectly regulate bone metabolism [7]. Dynamic muscle stimulation has been shown to generate intramedullar pressure (ImP) and low-level bone strain as a function of stimulation frequency. Induced dynamic ImP may ultimately enhance IFF in bone [8].

The patellar bone is located in the lower limb, and by being attached to the quadriceps femoris muscles it takes part in neutralizing forces during locomotion while the gravitational reaction forces probably are of minor im- 
portance since patella is not part of the axial skeleton.

Vasculature and supply of arterial blood flow are of vital importance for most tissues. Accordingly, blood supply is shown critical for bone metabolism, growth, and fracture healing. The total vascular pattern in irregular and flat bones departs significantly from the vascular organization of long bones, in that there is a considerably periosteal blood supply which is lacking in long bones [9].

The muscle pump hypothesis suggests that an arteriovenous pressure gradient is important for muscle perfusion [10]. The same process may also increase the hydraulic pressure in skeletal nutrient vessels and influence the bone capillary blood flow but also the capillary filtration [11]. In addition to the increased bone blood flow by muscle contractions different coupling factors between muscle contractions and fluid flow through bone exists [12]. The pressure waves generated from muscle pump contractions increase blood pressure during exercise by temporarily occluding arteries and veins leading to and from bone, and by increasing hydraulic pressure in bone capillaries [13]. Mechanical loading and vascular pressure have been proposed to drive IFF within the bone lacunar-canalicular system (LCS). Capillary pressure in the bone Haversian system thus could drive interstitial solute convection [14]. When bone is subjected to cyclic loading interstitial fluid drains from the LCS into and out of the vascular porosity that surrounds the bone capillaries [2]. In vivo tracer studies have demonstrated that vascular pressure is capable of driving IFF even in the absence of externally applied mechanical loading [15]. The cardiac contractions, and skeletal muscular contractions, induce peak shear stresses on the osteocyte cell processes [16]. However, it has also been shown that vascular pressure itself, does not enhance acute solute transport within the LCS, and subsequently its role in driving IFF is under debate [3].

A direct measurement of IFF or the bone capillary pressure is currently not possible. However, thanks to recent development we now have the possibility to measure pulsatile bone blood flow in the patellar bone [17]. Photoplethysmography (PPG) is a non-invasive optical technique for the measurement of changes in blood flow and has been used to monitor blood flow in skin and muscles [18-20], and lately also for hemodynamic sensing in implanted devices [21]. Furthermore, it has also been shown that changes in pulsatile blood flow within the patellar bone are possible to study continuously and non-invasively using the PPG technique $[17,22]$. PPG measurements might add information important for the understanding of which source of bone strain (muscle or vascular forces) but also which type of muscular work that predominate the adaptive stimulus in non-weight bearing flat bones.
The purpose of the present study was to describe reactive changes in pulsatile blood flow in the patellar bone after muscle contractions with two different work load intensities.

\section{MATERIALS AND METHODS}

\subsection{Subjects}

Forty-two healthy, regularly exercising, non-smoking, and normotensive individuals (14 women and 28 men) volunteered for the study, which was approved by the local ethic committee, (2007760-31/39). All subjects gave their written informed consent to participate in the study.

The mean age was 29 (range 20 to 53) years for the women and 37 (range 20 to 58) years for the men. The mean height was 171 (range 162 to 180 ) cm and 181 (range 165 to 192 ) $\mathrm{cm}$, for the women and men respectively. The weight among the women was 64 (range 55 to 73) $\mathrm{kg}$ and 83 (range 65 to 115) kg among the men.

The subjects were instructed to avoid exercises, other than normal activities of daily living, during the day the test was performed. All tests were executed in the afternoon. After 15 minutes of rest, the bone blood flow was recorded continuously from 3 minutes prior the intervention until 5 minutes after. One measurement was made on each leg.

\subsection{Muscle Contraction Exercises}

Dynamic isotonic leg extension/flexion exercises were performed in a leg extension machine (Nordic Gym 101SE, Caretaker Scand AB, Bollnäs, Sweden) with the purpose of utilizing low or high intensity muscle work in one leg respectively. The order of high intensity exercise based on 10 repetition maximum (RM), 10 RM $\sim 75 \%$ of $1 \mathrm{RM}$, and low intensity exercise based on $20 \mathrm{RM}, \sim 60 \%$ of $1 \mathrm{RM}$, was randomly performed on either the left or right leg. The high intensity work was performed continuously without any resting periods and the low intensity work with a two-second rest between the contractions. Work volume, expressed in kilograms totally lifted, was identical in both legs. A full isotonic leg extension and following knee flexion was performed during two seconds. The angle velocity was supervised by one of the authors by vocally counting the time. During the 15 minute resting period before exercising, the subjects were sitting in the leg extension machine with their legs supported and their knee joints in a resting position (20 degrees of flexion). This position was also held in the post-exercise period of 5 minutes. The time lap between the two measurements always exceeded $25 \mathrm{~min}$.

\subsection{Blood Flow Measurements}

A PPG probe was attached to the skin over the patella 
using adhesive tape [17]. In the PPG technique, light waves from a light-emitting diode is absorbed and scattered in the underlying tissue. The depth by which the light waves penetrate a tissue is primarily a function of wavelength and the optical geometry of the probe, but also of the optical qualities of the tissues of interest. In the patellar probe the wavelength of $804 \mathrm{~nm}$ was used with a distance between diode and the photodetector of $15 \mathrm{~mm}$. Variations in the photodetector signal are related to changes in blood flow [20].

A base-line, pre-exercise value was calculated from 60 seconds peak-to-peak recordings and a post-exercise value from 60 seconds peak-to-peak recordings when the reactive blood flow had reached a stable and steady-state level, Figure 1. This was mostly found within one minute after the intervention.

The PPG signal was analyzed using the software MATLAB, R2006b.

\subsection{Blood Pressure Measurements}

The blood pressure was measured continuously in twelve of the subjects by a non-invasive method (Finapress, Ohmeda 2300, Englewood USA). A small cuff was placed around the middle phalanx of the third finger on one hand that was placed on a padded platform at the level of the heart. A fast feedback loop including an infrared light source and detector, an air pump, and a microprocessor keeps the wall of the finger arteries at the so-called unloaded size, and the cuff pressure equals the arterial blood pressure. From the original pulse-wave signal the systolic and diastolic values were continuously determined. Mean systolic pressure was computed (MATLAB, R2006b) from peak systolic recordings during a 60-second, pre-exercise interval period, from a 10 -second interval at the end of the exercise where the highest systolic values could be seen, and from the 60 -second post-exercise interval during which the reactive bone blood flow measurements were made.

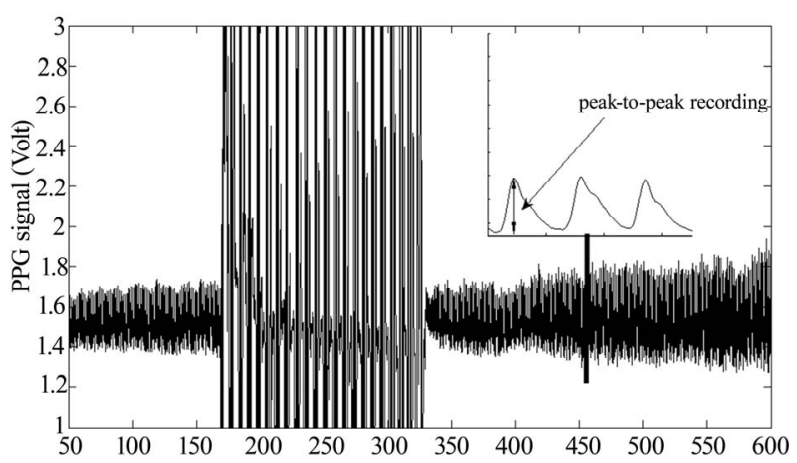

Figure 1. The PPG signal recordings of one subject from the patellar bone at rest, during, and after high intensity muscle work (10 RM).

\subsection{Statistics}

Mean values and ranges were calculated for quantitative, continuous data such as age, height, weight and systolic blood pressure. Non-normally distributed, continuous data were given as median values with inter quartile range (IQR) and the range from minimum to maximum. The dependent data of changes in measured blood flow were expressed as the percentage change post-exercise related to the pre-exercise level for each individual.

Wilcoxon's paired signed rank test was used to test the hypothesis of no difference between the two modes of muscle work, i.e. low and high intensity muscle work. The level of significance was set at $\mathrm{P}<0.05$. The statistical package Statistica 9.0 (StatSoft Inc., Tulsa, USA) was used for descriptive statistics and statistical analysis.

\section{RESULTS}

The individual responses of the paired raw data of measured bone blood flow in patella showed that the bone blood flow decreased among four individuals (10\%) while it was increased among the rest 38 individuals (90\%) after the high intensity muscle work, Figure 2(a). After the low intensity muscle work, the bone blood flow decreased in 12 (30\%), was unchanged in one (2\%) and increased in 28 (68\%) of the 41 reported measures, Figure 2(b).

\subsection{Change in Bone Blood Flow}

The high intensity muscle work induced by 10 RM, increased the measured bone blood flow with in median 61\% (IQR 120; range, -31 to 505). The low intensity muscle work induced by $20 \mathrm{RM}$ also resulted in an increase of patella bone blood flow with in median $22 \%$ (IQR 70; range, -52 to 155). However, high intensity muscle work induced a significantly higher blood flow change as compared to after the low intensity muscle work, $p=0.000073$ (Wilcoxon matched pairs signed rank sum test), Figure 3.

\subsection{Blood Pressure}

Mean systolic blood pressure (SBP) increased from 123.9 (range 101 to 137 ) before, to 158.5 (range 122 to 211) $\mathrm{mmHg}$ during the end of the high intensity muscle work, $n=42$, and from 121.9 (range 101 to 143) to 163.4 (range 139 to 198 ) $\mathrm{mmHg}$ during the end of the low intensity muscle work, $n=42$. During the post-exercise periods, simultaneously with the bone blood flow measurements, the mean SBP was 127.1 (range, 111 to 155 ) $\mathrm{mmHg}$ and 128.8 (range, 110 to 161) $\mathrm{mmHg}$ respectively. The patterns of SBP changes were in same range irrespective of the two levels of muscle work intensity induced. 


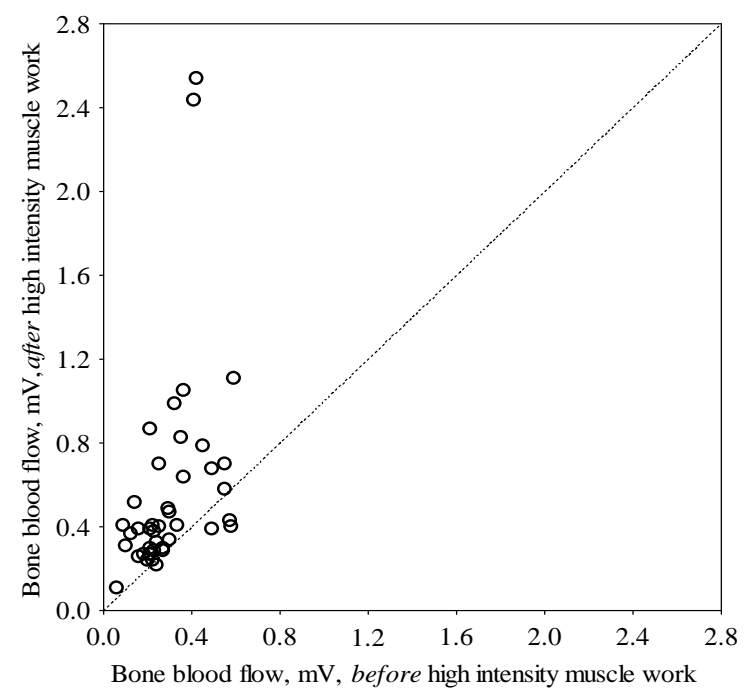

(a)

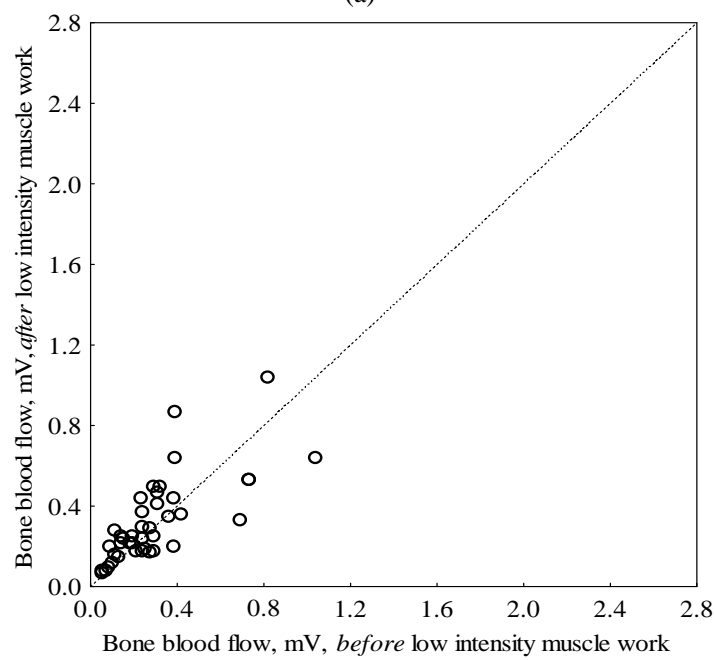

(b)

Figure. Paired raw data of measured patella bone blood flow using PPG signal ( $\mathrm{mV}$ ) before and after high intensity, $\mathrm{n}=42$ (a), and low intensity, $n=41$ (b), muscle work respectively.

\section{DISCUSSION}

To our knowledge, the present study is the first to quantify blood flow changes in human bone tissue in response to muscle contractions. We used a type of exercise often performed in muscle research, namely leg extension/ flexion exercises in a sitting position [23]. Our study indicates that pulsatile blood flow in the patellar bone is under the influence of contractions in the quadriceps femoris muscles, and exercise-induced bone hyperemia appears to be associated with the intensity of muscle contractions performed in muscles attached to the bone.

The patellar bone comprises a thin layer of cortical bone that surrounds a centre composed of trabecular bone but lacks a bone marrow cavity [24]. The intraosseous vessels in the patellar bone are encased in a rigid and

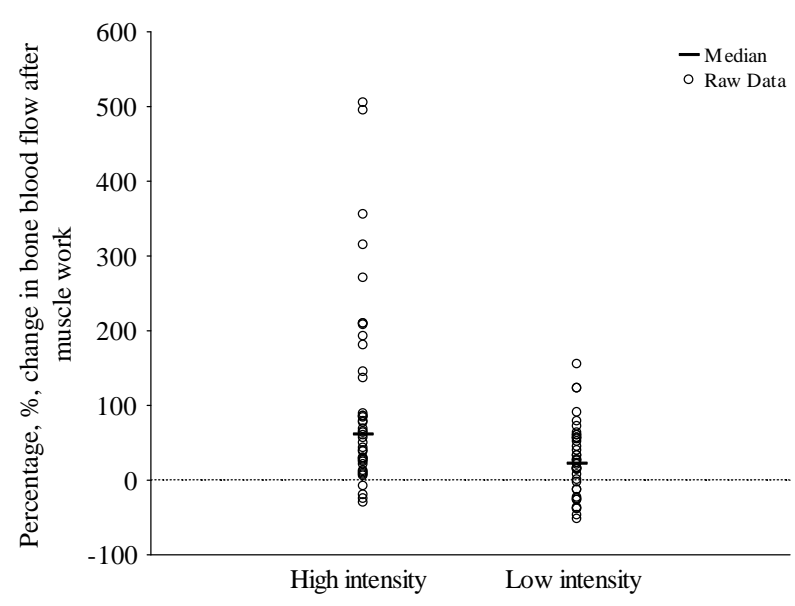

Figure 3. Percentage change in patella bone blood flow after high, $n=42$, and low intensity muscle work, $n=41$, respectively.

unyielding bony cylinder making the patellar bone suitable for PPG measurements. The pulse pressure could be transmitted directly to the sinusoids and venules across the extravascular fluid. It has been shown that the sinusoids, even in diaphyseal marrow, dilate and contract rhythmically, probably as a passive response to a pulsatile flow of blood in medullary arteries [25].

The question what source of bone strain predominates the adaptive stimulus is not easy to answer since the rate at which bone is exposed to strain can influence the adaptive response even to a greater extent than the absolute magnitude of the strain [3]. Despite the available data showing that bone responds to a variety of mechanical loads, many factors limit how to translate the evidence into effective practice. Obvious confounders include age- and sex-specific responses, along with nutrition, hormonal status, medications, and location of the bone within the skeleton. Also, we have to accurately define bone metabolism and take different aspects of skeletal adaptation into consideration; i.e. increased or reduced bone resorption and bone formation, periosteal or endosteal changes, but also whether bone mineral density (BMD) or bone mineral content (BMC) have been measured. Studies have shown, at least in men, that high-impact training and strength training produce site-specific increases in BMD due to bone formation [26]. While aerobic training (in this case most likely comparative to our low intensity muscle work) leads to changes indicating reduced bone resorption activity, anaerobic training (comparative to our high intensity muscle work) seems to result in an accelerated bone turn over. The impact of physical activity on bone turnover therefore may depend on the kind of exercised performed [27]. Notable, measurements of BMD are rarely performed in non-weight baring bones. 
It has been reported that muscle exercise hyperaemia is larger if the muscle work continues for a longer time at a level where aerobic metabolism is the prime source for energy demand [28]. The results of our study indicate a reversed reaction in bone tissue, i.e. the more intensive muscle work, the larger bone hyperaemia. There are two possibilities for the changes in bone blood flow seen in our study. Firstly, the proposed muscle pump mechanism is of different magnitudes in the two interventions used by us [11]. Leg extensions with a resistance of $10 \mathrm{RM}$ create a higher pressure in the muscle compartment than if the muscles contract against $20 \mathrm{RM}$ and this higher pressure might cause exercise hyperaemia in bone. Also, Recek [29] emphasized the importance of muscles as blood pumps. Theoretically, the pulsatile bone blood flow might be influenced by the SBP during the exercises since it is well-known that SBP rises during muscle activity. However, we found that the SBP was similarly changed, measured both at the end of the exercise periods and in the post-exercise periods, irrespectively of the type of muscle work performed. This indicates that at least the reactive pulsatile patellar bone blood flow is not primarily dependent on the SBP.

Secondly, in muscle tissue local hypoxia is known to be of great importance for peripheral blood flow even though most details of this process are still unknown. The metaboreflex, i.e. activated chemically sensitive nerves located in muscle parenchyma evoke increases in sympathetic activity, has been shown to regulate cardiac output, blood pressure and regional blood flow regulations [30]. Hypoxia is a possible regulator of microcirculation also in bone tissue. This has however not yet been studied in humans. Shim and Peterson [31] observed that the metabolic control mechanism was the most potent one regulating bone blood flow in rabbits. The blood flow appeared to be closely related to the oxygen and carbon dioxide tension, the $\mathrm{pH}$, and the acid metabolites in blood. Following a period of ischemia, the outflow venous volume increased two to three times in their study. Brookes \& Revell [9] suggested that local acidosis provides a stimulus for bone accumulation, and Gross et al. [32] hypothesized that hypoxia was responsible for the bone vasoregulation found in their study. These statements are in accordance with our results showing that high intensity muscle work increased the post exercise patellar blood flow $61 \%$. Gross et al. [32] showed that, during low and moderate levels of exercise (treadmill running) in dogs, the vascular resistance in axial bone increased two to fourfold, while an increased vasodilatation in adjacent muscles was found. Our study indicates a reversed reaction in the patellar bone. In contrast to us, Gross et al. [32] studied blood flow regulation during steady-state treadmill running and not post exercise hy- peraemia after resistant exercises.

The large bone hyperaemia seen in our study could serve other aspects of bone homeostasis than nutrition delivery. It has been proposed that an adaptive response to alternating venous pressure by the muscular pump mechanism could accelerate the IFF and thus impact bone metabolism. Exercise hyperaemia might induce reactions of vital importance for bone tissue such as increasing the IFF [12]. Interestingly, gravitational loaded bone models have shown that removing muscle loads will increase internal bone stresses during movement [12]. The fact that muscles both impart and neutralize forces on long bones during gait must be recognized when discussing muscle load on bone tissue. In cranial bones this situation is hardly present, but the patellar bone might resemble long bones in this aspect.

\subsection{Limitations and Strengths of the Study}

This is the first report of non-invasive bone blood flow measurement after muscle exercise in humans, and as such many limitations must be discussed. The most obvious limitation of the present PPG technique is the lack of a gold standard technique to compare it against. The patellar probe has been shown to discriminate between blood flow in the skin over the patella, and the patellar blood flow [17]. In a review by Allen [20], the validity and reliability of PPG measurements were discussed. For basal perfusion of the microcirculation the amplitude of the PPG pulse often correlated with laser Doppler blood flow (LDF). Recently, Hagblad et al. [33] showed that, combining PPG and LDF blood flow at different depth could be measured. Ultrasound measurements were used to differentiate the tissues beneath the probe and thus validate the capillary beds measured. A similar PPG probe as in our study was used to measure blood flow at a depth of $23 \mathrm{~mm}$, a distance well within the range of the patellar trabecular bone.

There are a limited number of studies quantifying the repeatability or reproducibility of PPG measurements. Jago and Murray [34] addressed the uncertainty in PPG measurements for a group of healthy adult subjects. An averaging period covering at least 60 heartbeats has been suggested to improve confidence in the single timing amplitude measurements extracted from the PPG pulse [20], and this time period was used in our study. Also, the PPG signal is calibrated before every new measurement and this will have impact on the magnitude of the PPG signal (voltage levels). Thus, results can only be analyzed as changes from one point in time to another. The same limitation is, however, present in studies using LDF [35] or NIRS [36].

Using a non-invasive technique to study blood flow has, however, many advantages. It is suitable for human 
studies and involves neither invasive manipulation of tissues nor their innervations, both which affect blood flow heterogeneity. The PPG technique also makes it possible to study blood flow continuously.

Since we do not know if the blood flow increase found is physically important, we cannot provide a power calculation. Are 42 subjects enough or does the variability seen in the measurements indicate that blood flow in bone tissue must be studied using larger cohorts? However, in studies on blood flow using other optical techniques, often less than 20 subjects has been included [35].

It has been suggested that $10-15$ minutes of rest is enough to down-regulate bone blood flow to base-line values [37]. We used the proposed resting time but, since this has not been extensively studied in humans, there might be a need for a much longer resting period, maybe hours. Also, it is possible that base-line levels of bone blood flow is influenced by auto-regulation and might therefore continually be changing during rest. The activity during the day before the measurement, locomotion, and posture maintenance could have a greater impact on resting bone blood flow than what has been reported. In the future, we eventually must control the pre-exercising circumstances more properly.

\section{CONCLUSIONS}

Resistance exercise induced patellar bone hyperaemia appears to be dependent of the intensity of muscle contractions in the quadriceps femoris muscle.

\section{CONFLICT OF INTEREST}

The authors declare no conflict of interest.

\section{REFERENCES}

[1] Piekarski, K. and Munro, M. (1977) Transport mechanism operating between blood supply and steocytes in long bones. Nature, 269, 80-82.

doi:10.1038/269080a0

[2] Fritton, S. and Weinbaum, S. (2009) Fluid and solute transport in bone: Flow-induced mechanotransduction. Annual Review of Fluid Mechanics, 41, 347-374. doi:10.1146/annurev.fluid.010908.165136

[3] Robling, A., Castillo, A. and Turner, C. (2006) Biomechanical and molecular regulation of bone remodeling. Annual Review of Biomedical Engineering, 8,455-498. doi:10.1146/annurev.bioeng.8.061505.095721

[4] Judex, S. and Carlson, K. (2009) Is bone's response to mechanical signals dominated by gravitational loading? Medicine and Science in Sports and Exercise, 41, 2037-2043. doi:10.1249/MSS.0b013e3181a8c6e5

[5] Gross, T., Poliachik, S., Prasad, J. and Bain, S. (2010) The effect of muscle dysfunction on bone mass and morphology. Journal of Musculoskeletal \& Neuronal Interactions, 10, 25-34.

[6] Frost, H. (2003) Bone’s mechanostat: A 2003 update. The
Anatomical Record. Part A, Discoveries in Molecular, Cellular, and Evolutionary Biology, 275, 1081-1101.

doi:10.1002/ar.a.10119

[7] Hamrick, M. (2010) Basic science and mechanisms of muscle-bone interactions. Journal of Musculoskeletal \& Neuronal Interactions, 10, 1-2.

[8] Qin, Y. and Lam, H. (2009) Intramedullary pressure and matrix strain induced by oscillatory skeletal muscle stimulation and its potential in adaptation. Journal of Biomechanics, 42, 140-145.

doi:10.1016/j.jbiomech.2008.10.018

[9] Brookes, M. and Revell, W.J. (1998) Blood supply of bone. Scientific aspects. Springer, London, 70-74, 291303.

[10] Laughlin, H. (2005) The muscle pump. What question do we want to answer? Journal of Applied Physiology, 99, 774. doi:10.1152/japplphysiol.00578.2005

[11] Winet, H. (2003) A bone fluid flow hypothesis for muscle pump-driven capillary filtration: II proposed role for exercise in erodible scaffold implant incorporation. European Cells \& Materials, 6, 1-11.

[12] Qin, Y., Lam, H., Ferreri, S. and Rubin, C. (2010) Dynamic skeletal muscle stimulation and its potential in bone adaptation. Journal of Musculoskeletal \& Neuronal Interactions, 10, 12-24.

[13] Laughlin, H. and Joyner, M. (2003) Closer to the edge? Contractions, pressures, waterfalls and blood flow to contracting skeletal muscle. Journal of Applied Physiology, 94, 3-5.

[14] Li, W., Gardinier, J., Price, C. and Wang, L. (2010) Does blood pressure enhance solute transport in bone lacunar-canalicular system? Bone, 47, 353-359. doi:10.1016/j.bone.2010.05.005

[15] Wang, L., Ciani, C., Doty, S. and Fritton, S. (2004) Delineating bone's interstitial fluid pathway in vivo. Bone, 34, 499-509. doi:10.1016/j.bone.2003.11.022

[16] Wang, L., Fritton, S., Weinbaum, S. and Cowin, S. (2003) On bone adaption due to venous stasis. Journal of Biomechanics, 36, 1439-1451. doi:10.1016/S0021-9290(03)00241-0

[17] Näslund, J., Lindberg, L., Lundeberg, T. and Linnarsson, D. (2006) Non-invasive continuous estimation of blood flow in human patella bone. Medical \& Biological Engineering \& Computing, 44, 501-509. doi:10.1007/s11517-006-0070-0

[18] Kamal, A., Harness, J., Irving, G. and Mearns, A. (1989) Skin photoplethysmography-A review. Computer Methods and Programs in Biomedicine, 28, 257-269. doi:10.1016/0169-2607(89)90159-4

[19] Zhang, Q., Lindberg, L., Kadefors, R. and Styf, J. (2001) A noninvasive measure of changes in blood flow of the human anterior tibial muscle. European Journal of Applied Physiology, 85, 567-571. doi:10.1007/s004210100496

[20] Allen, J. (2007) Photoplethysmography and its application in clinical physiological measurement. Physiological Measurement, 28, R1-39. doi:10.1088/0967-3334/28/3/R01

[21] Turcott, R. and Pavek, T. (2008) Hemodynamic sensing using subcutaneous photoplethysmography. American Journal of Physiology. Heart and Circulatory Physiology, 295, 2560-2572. doi:10.1152/ajpheart.00574.2008 
[22] Näslund, J., Waldén, M. and Lindberg, L. (2007) Decreased pulsatile blood flow in the patella in patellofemoral pain. American Journal of Sports Medicine, 35, 1668-1673. doi:10.1177/0363546507303115

[23] Polito, M., Farinattia, P., Lirad, V. and Nobregac, A. (2007) Blood pressure assessment during resistance exercise: Comparison between auscultation and finapres. Blood Pressure Monitoring, 12, 81-86. doi:10.1097/MBP.0b013e32809ef9f1

[24] Hughes, S., Cammarata, A., Steinmann, S. and Pellegrini, V. (1998) Effect of standard total knee arthroplasty surgical dissection on human patellar blood flow in vivo: an investigation using laser doppler flowmetry. Journal of the Southern Orthopaedic Association, 7, 198-204.

[25] Brånemark, P. (1959) Vital microscopy of bone marrow in the rabbit. Scandinavian Journal of Clinical and Laboratory Investigation. Supplementum, 11, 5-82.

[26] Banfi, G., Lombardi, G., Colombini, A. and Lippi, G. (2010) Bone metabolism markers in sports medicine. Sports Medicine (Auckland, N.Z.), 40, 697-714. doi:10.2165/11533090-000000000-00000

[27] Lester, M., Urso, M., Evans, R., Pierce, J., Spiering, B., Maresh, C., Hatfield, D., Kraemer, W. and Nindl, B. (2009) Influence of exercise mode and osteogenic index on bone biomarker responses during short-term physical training. Bone, 45, 768-776. doi:10.1016/j.bone.2009.06.001

[28] Kalliokoski, K., Kemppainen, J., Larmola, K., Takala, T., Peltoniemi, P., Oksanen, A., Ruotsalainen, U., Cobelli, C., Knuuti, J. and Nuutila, P. (2000) Muscle blood flow and flow heterogeneity during exercise studied with positron emission tomography in humans. European Journal of Applied Physiology, 83, 395-401. doi:10.1007/s004210000267

[29] Recek, C. (2010) Venous pressure gradients in the lower extremity and the hemodynamic consequences. VASA. Journal for vascular diseases, 39, 292-297.
[30] Boushel, R. (2010) Muscle metaboreflex control of the circulation during exercise. Acta Physiologica, 199, 367-383. doi:10.1111/j.1748-1716.2010.02133.x

[31] Shim, S. and Patterson, F. (1967) A direct method of qualitative study of bone blood circulation. Surgery, Gynecology \& Obstetrics, 125, 261-268.

[32] Gross, P., Heistad, D. and Marcus, M. (1979) Neurohumoral regulation of blood flow to bones and marrow. American Journal of Physiology, 237, 440-448.

[33] Hagblad, J., Lindberg, L., Kaisdotter-Andersson, A., Bergstrand, S., Lindgren, M., Ek-Folke, M. and Lindén, M. (2010) A technique based on laser Doppler flowmetry and photoplethysmography for simultaneously monitoring blood flow at different tissue depths. Medical \& Biological Engineering \& Computing, 48, 415-422. doi:10.1007/s11517-010-0577-2

[34] Jago, J. and Murray, A. (1988) Repeatability of peripheral pulse measurements on ears, fingers and toes using photoelectric plethysmography. Clinical Physics and Physiological Measurement, 9, 319-330. doi:10.1088/0143-0815/9/4/003

[35] Hempfing, A., Schoeniger, R., Koch, P., Bischel, O. and Thomsen, M. (2007) Patellar blood flow during knee arthroplasty surgical exposure: inoperative monitoring by laser Doppler flowmetry. Journal of Orthopaedic Research, 25, 1389-1394. doi:10.1002/jor.20416

[36] Mathieu, D. and Mani, R. (2007) A review of the clinical significance of tissue hypoxia measurements in lower extremity wound management. The International Journal of Lower Extremity Wounds, 6, 273-283. doi:10.1177/1534734607310299

[37] Colleran, N., Wilkerson, M., Bloomfield, S., Suva, L., Turner, R. and Delp, M. (2000) Alterations in skeletal perfusion with simulated microgravity: A possible mechanism for bone remodeling. Journal of Applied Physiology, 89, 1046-1054. 